\title{
Spatio-Spectral Characterization of Local Field Potentials in the Subthalamic Nucleus via Multitrack Microelectrode Recordings
}

\author{
I.Telkes $^{1}$, Student Member, IEEE, N.F.Ince ${ }^{1}$, Senior Member, IEEE, I. Onaran ${ }^{1}$, A. Abosch ${ }^{2}$
}

\begin{abstract}
Deep brain stimulation of the subthalamic nucleus (STN) is a highly effective treatment for motor symptoms of Parkinson's disease. However, precise intraoperative localization of STN remains a procedural challenge. In the present study, local field potentials (LFPs) were recorded from three tracks during microelectrode recording-based (MER) targeting of $\mathrm{STN}$, in five patients. The raw LFP data were preprocessed in original recording setup and then data quality was compared to data with common average derivation. The depth-frequency maps were generated according to preprocessing results for each patient and spectral characteristics of LFPs were explored at each depth across different tracks and different subjects. Spatio-spectral analysis of LFP was investigated to see whether LFP activity can be used for optimal track selection and STN border identification. Analysis show that monopolar derivation suffer from various artifacts and/or power line noise which makes the interpretation of target localization very difficult in most of the subjects. Unlikely, bipolar derivation helps to recover the neurological signals and investigation of signal characteristics. The frequency-vs-depth maps using a modified Welch periodogram with robust statistics, demonstrated that a median-based spectrum estimation approach eliminates outliers pretty well by preserving band-specific LFP activity. The results indicate that there is a clear oscillatory beta activity around $20 \mathrm{~Hz}$ in all subjects. $1 / f$ normalization reveals the high frequency oscillations (HFOs) between 200-to-350 $\mathrm{Hz}$ in two subjects. It's noted that the optimal track selection is not consistent with the track having highest beta band oscillations in two out of five subjects. In conclusion, microelectrodederived LFP recordings may provide an alternative approach to single unit activity (SUA)-based MER, for localizing the target STN borders during DBS surgery. Despite the small number of subjects, the present study adds to existing knowledge about LFP-based pathophysiology of PD and its target-based spectral activities.
\end{abstract}

\section{INTRODUCTION}

Deep brain stimulation (DBS) of the subthalamic nucleus (STN) is an effective therapy for the treatment of the motor symptoms of Parkinson's Disease (PD) [1]. DBS involves the surgical implantation of a quadripolar electrode into the

*Research supported by National Science Foundation, award CBET1343548.

1.N.F.I. is with the Biomedical Engineering Department, University of Houston, Texas, 77204-5060, USA, (corresponding author: (713) 743 4461; e-mail: nfince@uh.edu). Senior IEEE Member

1.I.T. is with the Biomedical Engineering Department, University of Houston, Texas, 77204-5060, USA, (e-mail: itelkes@uh.edu).

1. I.O. is with the Biomedical Engineering Department, University of Houston, Texas, 77204-5060, USA, (e-mail: onaran@gmail.com).

2.A.A. is with the Department of Neurosurgery, Director of Stereotactic and Epilepsy Surgery, Director of Neurosurgery Research, University of Colorado, Aurora, CO 80045, USA (e-mail: aviva.abosch@ucdenver.edu)

U.S. Government work not protected by U.S. copyright motor territory of STN, followed by chronic stimulation via connection of the electrode to an implanted pulse generator (IPG). An important factor contributing to the efficacy of DBS is the accurate localization of STN in the brain. The small volume of STN motor territory, its depth from the cortical surface, its proximity to other critical neural structures, and the great variability of the STN location between subjects make precise targeting crucial as well as challenging [2]. Together with stereotactic imaging, intraoperative microelectrode recording (MER) is the most commonly used physiological technique to determine STN location for chronic implantation of DBS macroelectrode [3].

In MER-SUA, the electrical activity resulting from individual neurons is recorded by high impedance microelectrode tips. The electrophysiological signals are converted into audio and visual signals and interpreted by neurophysiologists or specialized neurosurgeons in order to localize the anatomical borders of STN [4]. The initial trajectory and stereotactic coordinates of the target are determined based on preoperative MRI and/or CT images. Microelectrodes are then inserted through cannulas into the brain and SUAs are recorded [5]. Following MER target localization, microelectrodes are withdrawn and replaced by the DBS macroelectrode. Although MER-SUA provides useful information for guiding surgery, there are several limitations of the technique. Interpretation of signal patterns to localize the anatomical borders of STN is subjective approach makes the procedure more open to human error, especially in the multi-target cases requiring complex interpretation [4]. It increases the surgery time and introduces the risk of intracranial hemorrhage. From a technical point of view, the spiking characteristics of single neurons around the region of STN is highly overlapping between different structures [6]. SUA recorded from the tip covers an activity only from a very small region. Its highly sparse and sensitive nature to noise reduces the identification of STN.

Unlike MER-SUA, local field potentials recorded from a larger electrode contact represent the aggregate activity of neuronal populations [7]. Therefore, they are particularly sensitive to synchronous and oscillatory firing patterns which makes them a big candidate for the target localization in motor disorders [10]. In PD, LFP recordings from STN are an important indicator of neural rhythms [8]. Studies have demonstrated an excessive synchrony in beta band (13$30 \mathrm{~Hz}$ ) activity in STN [9]. 
The aim of the present study was to explore the spatiospectral characterization of LFPs recorded from microelectrodes, in order to identify the anatomical borders of STN in DBS surgery. The less number of challenges in MER-LFP recordings in the operating room can reduce the surgery time and serve as a useful tool for target validation. It can also serve to further understand the neurophysiopathological characteristics of STN in PD.

\section{METHODS}

\section{A. Patients and surgery}

Five patients provided informed consent and with approval of the University of Minnesota Institutional Review Board were enrolled in this study. All study subjects had a diagnosis of idiopathic PD and exhibited typical Parkinsonian motor symptoms despite optimal medial therapy. All patients discontinued Parkinson's medications 12 hours prior to surgery. Per standard clinical routine, three simultaneous MER-SUA recording tracks were performed in each subject using local anesthetic alone in order to localize the target by using physiological signals. The initial target and trajectory were identified by stereotactic MRI fused to a stereotactic CT on a neuronavigational platform (StealthStation, Medtronic Corp, CO). Before the unilateral implantation of DBS electrode into target, microelectrode implantation into STN and simultaneous SUA recordings were obtained using a Neurodrive and Microguide system (AlphaOmega Inc., USA) respectively (Fig.1.).

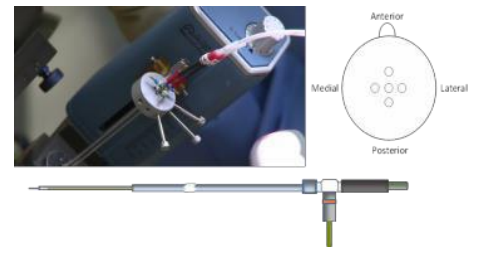

Fig.1.A schematic of the single channel microelectrode currently used in clinical practice with a bengun used to implant up to 5 microelectrodes.

\section{B. Recordings}

STN LFPs were recorded with XLTEK-EMU128FS system (Natus, San Carlos, California). Each microelectrode was inserted into brain passing through the cannula starting from $25 \mathrm{~mm}$ above the target continued until the electrode caught an excessive beta activity which is mostly around 0 to- $1 \mathrm{~mm}$ below target. The step size for each recording was $1 \mathrm{~mm}$ until $10 \mathrm{~mm}$ above the estimated target and then reduced to $0.5 \mathrm{~mm}$ all the way down. LFP data were recorded from the LFP contact which is $3 \mathrm{~mm}$ above the electrode tip along with EKG signal for 30 seconds at each depth. Signals were sampled at $2 \mathrm{kHz}$ with 16 bit A/D resolution. All raw data channels were high-pass filtered at $0.1 \mathrm{~Hz}$. Signals were transferred into a PC for off-line spectral analysis.

\section{Analysis}

Recorded LFP data were annotated and visualized to distinguish artifact and determine epochs of resting, active, and passive movements in the XLTEK system and then exported into MATLAB (Mathworks, Natick, Massachusetts) for processing. LFP data from all tracks were low-pass filtered using an FIR filter with a 450-Hz cutoff frequency, and then down-sampled to $1000 \mathrm{~Hz}$ for analysis to be able to scan a broad band of activity [8]. To further eliminate the EKG-based artifacts or other environmental factors, signals were filtered with a $2.5 \mathrm{~Hz}$ high-pass filter and $60 \mathrm{~Hz}$ notch filter at its harmonics.

During processing of LFP data, down-sampled signals were converted into bipolar derivations and compared to monopolar recordings. Because LFPs were recorded referenced to cannula in the OR recording setup, signal itself was used in monopolar configuration. For the bipolar derivation, mean of the three tracks were subtracted from each track itself to eliminate the common activity from channels. The weights of each track in monopolar and bipolar derivation can be seen in Table-I. In the bipolar derivation, common activity, which can be artifact or a long-lasting low band activity, on the channel is eliminated, on the other hand an undesired, small amount of signal from other tracks is introduced onto channel as well.

Table-I Weights of each track in monopolar and bipolar derivation

\begin{tabular}{|c|c|c|c|}
\hline & Track-1 & Track-2 & Track-3 \\
\hline MonoPolar & 1 & 0 & 0 \\
\hline Bipolar & 0.66 & -0.33 & -0.33 \\
\hline
\end{tabular}

In order to explore the frequency content of the LFP data at each depth, a depth-frequency analysis was generated similar to the well-published time-frequency analysis. It was observed that the LFP data were corrupted by many factors including tremor and/or environmental factors in the operating room setting despite the prior artifact removal methods. Therefore, the LFP spectrum with a modified Welch periodogram method was computed, including robust statistics. Simply, rather than using an average over different segments, a median operator to compute the periodogram which suppressed outliers was used. Specifically, for spectrum analysis, the fast Fourier transform (FFT) was computed with a 512 samples long Hanning window and the window was shifted with $50 \%$ overlap. After computing the squared magnitude in each sliding window, we used the median operator to estimate the LFP spectrum. We repeated this same procedure at each depth and the resulting spectra were used to visualize dynamic frequency spectrum of the LFP data on each track.

The 1/f spectral characteristic of many electrophysiological signals makes the visualization of high frequency band activity difficult due to decreasing log power with increasing frequency. Fig.2. shows the $1 / f$ nature of LFPs pointing that signal property at lower bands with higher power might be masking the high frequency activity with smaller power. In order to reveal the activity at higher bands, spectra representing the activity in all depths on each track were averaged to obtain a mean spectrum of three tracks, which was trend-fitted with an exponential curve. The inverse of this fitting curve was then multiplied, point by point, to the single-depth spectra for each track 


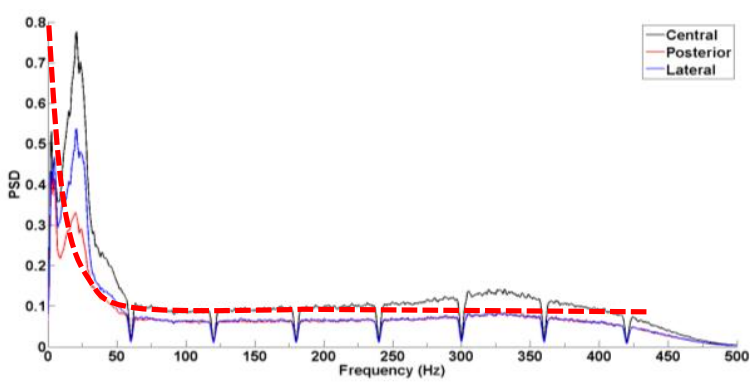

Fig.2. The spectrum of LFPs recorded from three tracks during microelectrode recordings shows the $1 /$ f characteristics of LFPs. Red dashed line shows the fitting curve.

independently. All of the depth-frequency maps were smoothed by using a Gaussian filter in order to increase visualization quality.

\section{RESULTS}

The raw LFP data of a representative subject is shown in Fig. 3.A. The high amount of artifacts which may be resulted from abrupt movements of the patient, other environmental factors, or effect of cannula can be seen in each track in monopolar derivation (Fig.1.A). When the common average method is applied into the same dataset, in Fig.3.B, it is clearly seen that all these artifacts are removed at various depths. Artifact-free LFP plots show that after the electrode reached a certain depth, high amplitude LFP activity was observed. This amplitude change occurred consistently in all subjects between the superior and inferior border of STN as identified by MER-SUA.

Fig.4. shows the frequency content of LFP activity at various depths in each track for the same subject. In Fig.4.A, it is evidently seen that monopolar derivation with full of artifacts make the interpretation of target localization very difficult. These artifacts are highly influenced by the $1 / f$ normalization due to their high amplitude nature. Hereby, it kills the advantageous of using normalized depth-frequency maps in monopolar derivation (not shown).

Fig.5. demonstrates the advantage of using bipolar derivation in depth-frequency maps in another subject. In the first row (A), monopolar maps show that it's very difficult to localize the STN based on excessive beta oscillation while bipolar maps (B) indicate pronounced, highly localized beta activity on the second track. The beta band information gets lost in the monopolar normalized maps because of the common activity at the low band all over the depths. Unlikely, the $1 / f$ normalization in bipolar derivation elicits the low amplitude activity at the high frequency bands by keeping the oscillatory activity at lower frequencies as it is.

Fig.6. indicates the different spectral characteristics of LFPs at various depths. Fig.6.A shows a strong $20 \mathrm{~Hz}$ activity below the MER-SUA determined superior border of STN. A weaker, at first gland, high gamma activity is noted earlier than the excessive beta oscillations in this subject.

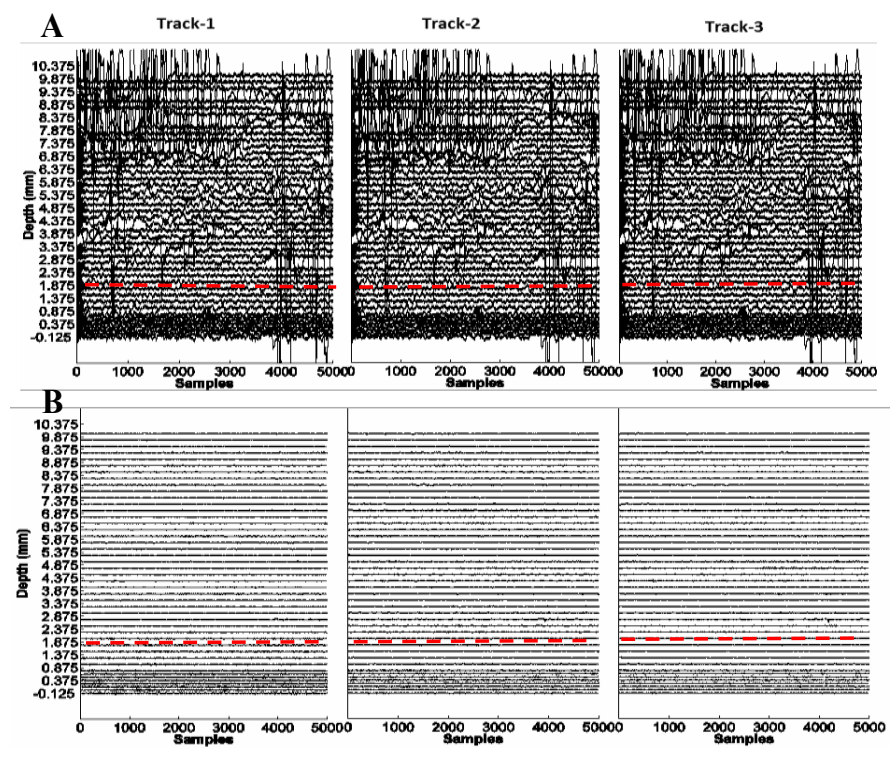

Fig.3. (A) The three tracks of raw LFP data in monopolar configuration for a representative subject. (B) The three tracks of raw LFP data in bipolar configuration for a representative subject.
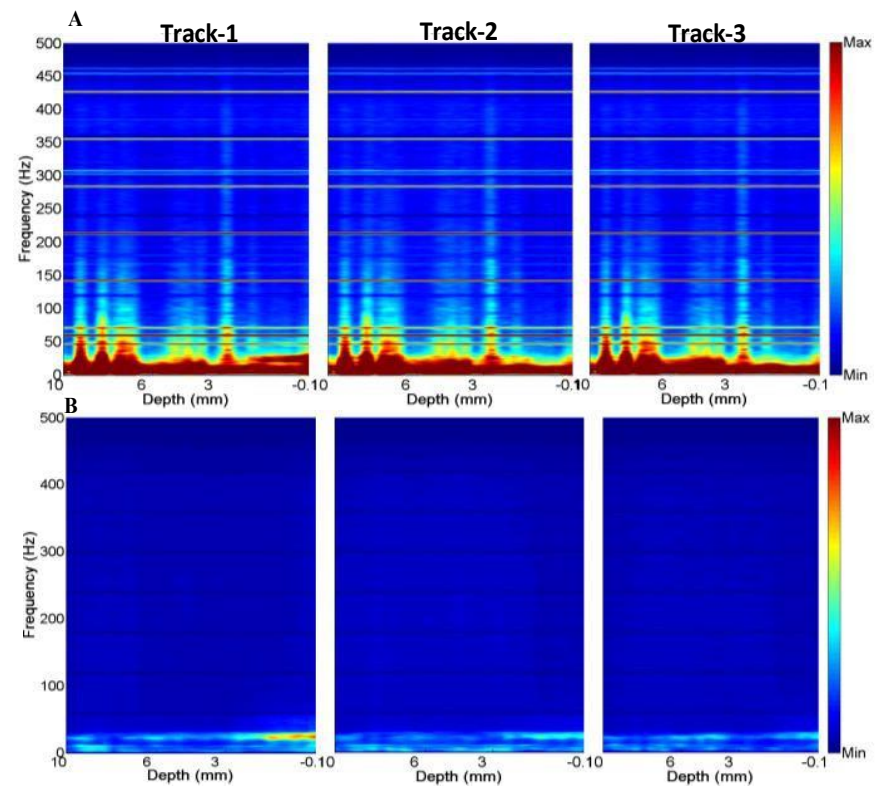

Fig.4. (A) Depth-frequency maps of monopolar LFP data for each track. (B) Depth-frequency maps of bipolar LFP data for each track.

When the $1 / f$ normalization is applied to the same dataset, both beta and high gamma oscillations and their spatial dynamics are clearly observed in Fig.6.C. On the other hand, in another patient, a totally different spatio-spectral dynamics are found. Fig.6.B shows a strong beta activity around $20 \mathrm{~Hz}$ up to $30 \mathrm{~Hz}$. It's difficult to visualize the high frequency oscillations in the same map. Yet, normalization 

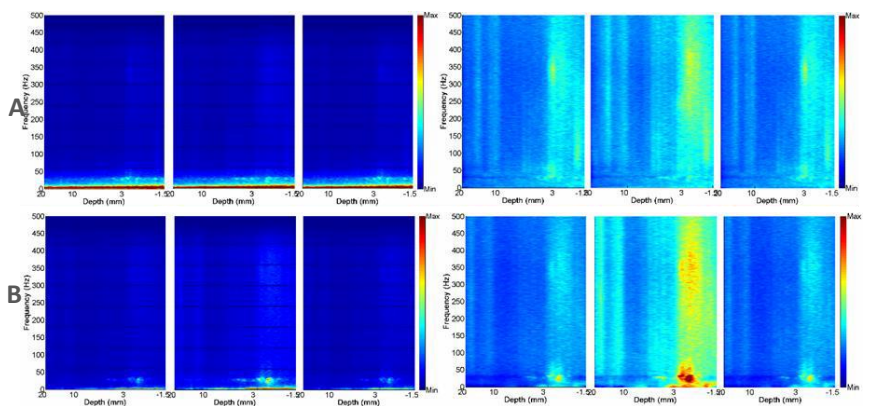

Fig.5. (A) Depth-frequency maps of monopolar LFP data for each track and its normalized map. (B) Depth-frequency maps of bipolar LFP data for each track and its normalized map
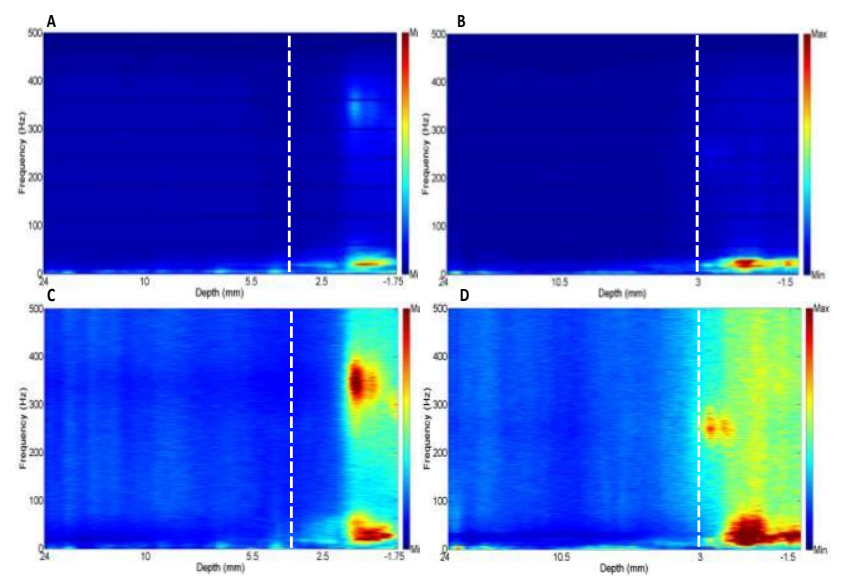

Fig.6. (A-B) Depth-frequency maps of bipolar LFP data of two subjects for selected track. (C-D) Normalized depthfrequency maps of bipolar LFP data of the same subjects for the same track.

technique elegantly displays the high gamma oscillations. Unlikely, the HFOs in this subject are less powerful and they are noted around $250 \mathrm{~Hz}$ with a more distinct temporal dynamics.

\section{DISCUSSION}

Previous studies suggested that the excessive beta-band activity of LFP can be used to localize STN [7]. In the present study, during a DBS electrode implantation surgery, we recorded LFPs from three tracks of microelectrodes in five PD subjects. We observed that analysis on data with recording setup cannot provide useful information because of the high influence of artifacts. However, the only limitation is not necessarily the artifact but also the masking effect of common activity at various depths which kills the localized oscillation specific to certain depths.

The visualization of raw LFP signals indicated increased LFP activity between the superior and inferior borders of the STN. After computing the LFP spectrum at each depth, we observed that excessive activity occurs not only in the betaband, but also in the higher bands, ranging from 40 up to $450 \mathrm{~Hz}$. The most interesting findings are the spectral and spatial dynamics of HFOs in two subjects. These dynamics are totally different from each other that it might be related to different structures. However they are both noted below the superior border of the STN based on MERSUA and they are both at the selected tracks. It is striking that in the other two subjects high beta oscillations are observed in the track rather than the selected one. Plus, these subjects do not show any significant HFOs even in the normalized maps. Our results support the possibility that there is a depth-related difference between subjects in spectral scenery. Such differences might account for the observed variability in long-term symptom suppression in patients with PD treated with DBS. The findings presented here might contribute to further investigation of pathophysiology of PD from a spatio-spectral perspective.

Due to the more robust nature of the LFP signal, instead of single neurons, LFP signal based confirmation of DBS location might be more advantageous than MER-SUA. LFPbased DBS surgery might also lend itself to a more automated approach to interpreting complex intraoperative neurophysiology rather than the current scenario that requires significant expertise in auditory MER-SUA.

\section{ACKNOWLEDGMENT}

This research was supported by National Science Foundation, award CBET1343548.

\section{REFERENCES}

H. Cagnan, K. Dolan, X. He, M. F. Contarino, R. Schuurman, P. van den Munckhof, W. J. Wadman, L. Bour, and H. C. F. Martens, "Automatic subthalamic nucleus detection from microelectrode recordings based on noise level and neuronal activity.," J. Neural Eng., vol. 8, no. 4, p. 046006, Aug. 2011.

[2] P. Krack, M. I. Hariz, C. Baunez, J. Guridi, and J. A. Obeso, "Deep brain stimulation: from neurology to psychiatry?," Trends Neurosci., vol. 33, no. 10, pp. 474-484, 2010.

[3] A. Zaidel, A. Spivak, L. Shpigelman, H. Bergman, and Z. Israel, "Delimiting subterritories of the human subthalamic nucleus by means of microelectrode recordings and a Hidden Markov Model.," Mov. Disord., vol. 24, no. 12, pp. 1785-93, Sep. 2009.

[4] T. A., "An automated navigation system for deep brain stimulator placement using hidden Markov models," Neurosurgery.,vol. 10.1227/01, pp. 108-17, 2010.

[5] K. P. Michmizos, G. L. Tagaris, D. E. Sakas, K. S. Nikita, and S Member, "Automatic Intra-Operative Localization of STN using the Beta Band Frequencies of Microelectrode Recordings," pp.1-6.

[6] Wong, S., et al. "Functional localization and visualization of the subthalamic nucleus from microelectrode recordings acquired during DBS surgery with unsupervised machine learning." J. Neural Eng.,vol. 6.2,, p.026006, 2009

[7] C. C. Chen, A. Pogosyan, L. U. Zrinzo, S. Tisch, P. Limousin, K. Ashkan, T. Yousry, M. I. Hariz, and P. Brown, "Intra-operative recordings of local field potentials can help localize the subthalamic nucleus in Parkinson's disease surgery.," Exp. Neurol., vol. 198, no. 1, pp. 214-21, Mar. 2006.

N. F. Ince, A. Gupte, T. Wichmann, J. Ashe, T. Henry, M. Bebler, L. Eberly, and A. Abosch, "Selection of optimal programming contacts based on local field potential recordings from subthalamic nucleus in patients with Parkinson's disease.," Neurosurgery, vol. 67, no. 2, pp. 390-7, Aug. 2010.

[9] N. Jenkinson and P. Brown, "New insights into the relationship between dopamine, beta oscillations and motor function.," Trends Neurosci., vol. 34, no. 12, pp. 611-8, Dec. 2011.

[10] A. Priori, G. Foffani, A. Pesenti et al., 'Rhythm-specific pharmacological modulation of subthalamic activity in Parkinson's disease", Exp. Neurol., vol. 189, no. 2, pp. 369-379, 2004. 